\title{
Comparison of the Impact of Breathing Strengthening Exercises and Balloon Blowing Training on the Pulmonary Function of Elderly Smokers
}

\begin{abstract}
The purpose of this study compared the ability of feedback breathing training (FBT) and balloon blowing training to enhance the breathing of elderly people. The subjects were randomly and evenly divided into a feedback breathing training group (FBTG) and a balloon blowing training group (BBTG). Each group trained 3 times a week for 4 weeks, with the training suspended during the last 2 weeks. Pulmonary function measurements were obtained before the test and 2, 4 and 6 weeks after the test: forced vital capacity (FVC), forced expiratory volume at one second (FEV1), FEV1/FVC, peak expiratory flow (PEF) and vital capacity (VC). A repeatedmeasures ANOVA was conducted for the significance test. The FBT resulted in a significant increase in the FVC, FEV1/FVC, PEF, and VC of the elderly smokers after 4 weeks and a significant decrease in the FVC, FEV1/FVC, and PEF after 6 weeks. The BBT resulted in a significant increase in the FVC, FEV1, FEV1/FVC, PEF, and VC of the elderly smokers after 4 weeks and a significant decrease in the FVC, FEV1/FVC, and PEF after 6 weeks. In conclusion, An at home breathing rehabilitation program, in addition to balloon blowing, could increase the breathing performance of elderly people.
\end{abstract}

Key words: Feedback Breathing Training; Balloon Blowing Training; Elderly

\author{
Hyun ju Jun ${ }^{\mathrm{a}}$, Sang Wan Lim ${ }^{\mathrm{b}}, \mathrm{Ki}$ \\ Jong $\mathrm{Kim}^{\mathrm{a}}$ \\ ${ }^{a}$ Cheongam College, Suncheon; ' ${ }^{\text {P Pohang }}$ \\ College, Pohang, Korea \\ Received : 11 June 2015 \\ Revised : 22 July 2015 \\ Accepted : 11 August 2015 \\ Address for correspondence \\ Ki Jong Kim, PT. Ph.D \\ Department of Physical Therapy, \\ Cheongam College, 1641 Noksaek-ro, \\ Suncheon, Korea \\ Tel: 82-61-740-7339 \\ E-mail: kjparadise@hanmail.net
}

\section{INTRODUCTION}

The aged population is continuously increasing due to economic growth and advancements in medical techniques(1). In older individuals, strength exercises are important to be able to perform independent activities of daily living. Strength exercises for people over 60 years should consider their structural and functional adaptation and physiological elasticity $(2,3)$.

With aging, the stiffness of the chest wall increases, and respiratory muscular strength decreases, giving rise to pathological problems and respiratory system disease. Such changes cause breathing problems, as well as ventilation and perfusion imbalance $(4,5)$. Decreased cardiopulmonary function among elderly people can also influence the amount of muscle decrease and the extent of physical disabilities(6).

The level of carbon monoxide is increased in the blood of smokers, whereas the oxygen carrying capacity of the blood is decreased. As a result, cell oxygen is insufficient, which has an adverse impact on their ability to perform exercise(7). Smoking is one of the major causes of early death $(8,9)$. It is known to influence not only the respiratory tract but also the whole body(10). Smoking is a risk factor for the pulmonary function of respiratory disease, having a negative impact on breathing(11).

Breathing problems can be managed using stable chest exercises, which are aimed at strengthening the respiratory muscle and improving breathing patterns(12). Feedback breathing training using SPIRO TIGER ${ }^{\circledR}$ has been reported to increase endurance and improve people's quality of life(13, 14). Balloon blowing has also been reported to be effective in increasing breathing muscular strength and breathing ability(15).

Most previous studies of breathing muscular strength training involved patients with respiratory system or nervous system diseases. Research 
on systematic breathing training is scarce(16). Recently, muscle training has received an increasing amount of attention as a means of improving respiratory muscle function and cardio respiratory endurance(12).

Elderly patients with respiratory disease can experience breathing problems due to the increased morbidity associated with the disease. Breathing muscular strength training is important for such patients. The present study compared the ability of feedback breathing training(FBT) and balloon blowing training(BBT) to enhance the breathing of elderly people.

\section{METHODS}

\section{Subjects}

The study groups consisted of 20 smokers older than 65 years who attended a welfare center in $\mathrm{M}$ City, Jeollanam-do, Korea. The subjects were randomly and evenly divided into a feedback breathing training group (FBTG) and a balloon blowing training group (BBTG). Each group trained three times a week for 4 weeks, with the training suspended during the last 2 weeks. As the study groups consisted of elderly individuals, pain in the heart and dizziness symptoms were carefully monitored, and sufficient resting was given for conditioning.

This study was approved by the Bioethics Council of Dongshin University (1040708-201406-BM001-02). The subjects understood the purpose of the study and volunteered to participate in the study. The general characteristics of the study subjects are described in Table 1.

Table 1. General characterstic of subjects

\begin{tabular}{ccc}
\hline & FBTG $(\mathrm{n}=10)$ & BBTG $(\mathrm{n}=10)$ \\
\hline Age(years) & $72.60 \pm 9.0$ & $70.00 \pm 3.5$ \\
Height(cm) & $163.20 \pm 6.39$ & $152.69 \pm 6.33$ \\
Weight(kg) & $66.64 \pm 9.87$ & $59.45 \pm 9.58$ \\
Sex(male/female) & $8 / 2$ & $7 / 3$ \\
\hline
\end{tabular}

\section{Measurement Tools and Measuring Methods}

In the FBTG, the participants trained for $15 \mathrm{~min}$ using a SPIRO TIGER ${ }^{\circledR}$ device. In the BBTG, the participants maintained one set for $1 \mathrm{~min}$, and each set was repeated 3 times. A round-type balloon that was comfortable to hold and blow was used in the BBTG. The breathing training program was implemented 3 times a week for 4 weeks. A rotation exercise consisting of abdominal breathing and body trunk was used to relax the muscle tone before starting the training.

Pulmonary function was measured in a sitting posture in a chair using the CardioTouch 3000S (BIONET, Korea). The following pulmonary function measurements were obtained before the test and 2, 4, and 6 weeks after the test: forced vital capacity (FVC), forced expiratory volume at one second (FEV1), FEV1/FVC, peak expiratory flow $(\mathrm{PEF})$ and vital capacity (VC). The values were measured three times, and the highest value was used(17).

\section{Data Analysis}

Predictive Analytics Software (PASW), version 18.0 was used for the analysis of the data. A repeated measures ANOVA was conducted for the significance test of FBTG and BBTG by time. A post hoc Bonferroni test was implemented. In cases where there was a significant between-group difference by group and measurement time, a repeated contrast test was performed for each group. The significance level was set at .05 for all data.

\section{RESULTS}

According to the repeated contrast test results of the measurement time in each FVC group (Table 2), FBTG after 2 weeks showed no significant difference ( $p>05)$. However, there was a significant difference in the FEV of the FBTG after 4 and 6 weeks ( $p<.05$ for both). BBTG after 2 weeks showed no significant difference (p $>$.05), BBTG after 4 weeks showed a significant difference (p<.05), and BBTG after 6 weeks also showed a significant difference $(\mathrm{p}<.05)$.

According to the repeated contrast test results of the measurement time in each FEV1 group (Table 2 ), the FBTG showed no significant difference after 2 weeks ( $p>05)$, 4 weeks ( $p>.05)$, or 6 weeks (p).05). In the BBTG, there was no significant dif- 
ference in the FEV1 after 2 weeks (p>.05) or 6 weeks ((p).05), but there was a significant difference after 4 weeks $(p<.05)$.

According to the repeated contrast test results of the measurement time in each FEV1/FVC group (Table 2), the FBTG after 2 weeks showed no significant difference (p〉.05), the FBTG after 4 weeks showed a significant difference ( $p<.05)$, and the FBTG after 6 weeks also showed a significant difference $(p<.05)$. The BBTG showed no significant difference after 2 weeks ( $p>05)$, the BBTG showed a significant difference after 4 weeks ( $\mathrm{p}<.05)$, and the BBTG showed a significant difference after 6 weeks $(\mathrm{p}<.05)$.

Table 2. Changes of pulmonary function in smokers groups

\begin{tabular}{ccccccccc}
\hline \multirow{2}{*}{$\begin{array}{c}\text { Pulmonary } \\
\text { function }\end{array}$} & \multicolumn{2}{c}{ Pre } & \multicolumn{2}{c}{2 weeks } & \multicolumn{2}{c}{4 weeks } & \multicolumn{2}{c}{6 weeks } \\
\cline { 2 - 8 } & FBTG & BBTG & FBTG & BBTG & FBTG & BBTG & FBTG & BBTG \\
\hline FVC** & $3.40 \pm .18$ & $3.26 \pm .33$ & $3.44 \pm .24$ & $3.46 \pm .61$ & $3.77 \pm .30^{*}$ & $3.89 \pm .50^{*}$ & $3.66 \pm .20^{*}$ & $3.64 \pm 41^{*}$ \\
FEV1 & $2.68 \pm .54$ & $2.54 \pm .60$ & $2.70 \pm .55$ & $2.69 \pm .46$ & $2.80 \pm .61$ & $3.13 \pm .54^{*}$ & $2.71 \pm .52$ & $2.78 \pm 47$ \\
FEV1 / FVC & $69.90 \pm 9.50$ & $73.00 \pm 9.07$ & $69.90 \pm 9.80$ & $73.10 \pm 9.50$ & $74.10 \pm 9.35^{*}$ & $76.10 \pm 9.61^{*}$ & $69.90 \pm 8.97^{*}$ & $73.30 \pm 10.50^{*}$ \\
PEF & $4.20 \pm 1.38$ & $3.40 \pm 1.16$ & $4.20 \pm 1.39$ & $3.40 \pm 1.19$ & $4.23 \pm 1.39^{*}$ & $3.64 \pm 1.24^{*}$ & $4.20 \pm 1.39^{*}$ & $3.53 \pm 1.23^{*}$ \\
VC & $2.95 \pm .43$ & $2.98 \pm .37$ & $2.95 \pm .43$ & $2.99 \pm .37$ & $3.32 \pm .49^{*}$ & $3.31 \pm .30^{*}$ & $3.22 \pm .51$ & $3.23 \pm 40$ \\
\hline
\end{tabular}

Values are showed mean \pm SD

Significance was tested by repeated-ANOVA(* : p<.05)

Post-hoc was tested by Bonferroni(** : p<.05)

FVC : Forced Vital Capacity

FEV1: Forced Expiratory Volume at one second

PEF : Peak Expiratory Flow

VC : Vital Capacity

FBTG : Feedback Breathing Training Group

BBTG : Ballon-Blowing Training Group

According to the repeated contrast test results of the measurement time in each PEF group (Table 2 ), the FBTG showed no significant difference after 2 weeks ( $>$.05), the FBTG showed a significant difference after 4 weeks $(p<.05)$, and the FBTG showed a significant difference after 6 weeks ( $p<.05)$. The BBTG showed no significant difference after 2 weeks ( $p>05)$, the BBTG showed a significant difference after 4 weeks $(p<.05)$, and the BBTG also showed a significant difference after 6 weeks ( $p<.05)$.

According to the repeated contrast test results of the measurement time in each VC group (Table 2), the FBTG after 2 weeks showed no significant difference (p $\rangle .05)$, the FBTG after 4 weeks showed a significant difference ( $p<.05)$, and the FBTG after 6 weeks also showed no significant difference (p>.05). The BBTG after 2 weeks showed no significant difference (p>.05), the BBTG after 4 weeks showed a significant difference $(p<.05)$, and the BBTG after 6weeks showed no significant difference ( $p>$.05).

\section{DISCUSSION}

This study applied breathing training to elderly smokers for 4 weeks and measured their pulmonary function 6 weeks after suspending the training to investigate the effects of the breathing training and the sustainability of these effects.

Smoking is closely related to chronic respiratory system disease, and pulmonary function tests are important in the diagnosis and treatment of lung disease. A negative correlation between decreased pulmonary function and age has been reported(17). This supports the ground for comparing the effects on pulmonary function of the smoking elderly in this study.

The FBT resulted in a significant increase in the FVC, FEV1/FVC, PEF, and VC of the elderly smokers after 4 weeks and a significant decrease in the FVC, FEV1/FVC, and PEF after 6 weeks. On balance, the effects of the training persisted 6 weeks after suspending the training only in the case of the VC. 
In pulmonary function measurements of patients with chronic obstructive pulmonary disease (COPD) who took part in FBT, the FEV1 and FEV1/FVC increased in a sitting position compared to a supine position(18). Decreased perception of dyspnea was also reported in these patients(13). A 30 min FBT program three times a week for 4 weeks resulted in a statistically significant increase in the FVC, FEV1, PEF, and VC of 45 patients with restrictive pulmonary disease(19). Based on the current literature, FBT is an effective treatment for elderly smokers with obstructive and restrictive pulmonary disease, and the position in which pulmonary function measurements are made is important.

In the present study, the BBT resulted in a significant increase in the FVC, FEV1, FEV1/FVC, $\mathrm{PEF}$, and $\mathrm{VC}$ of the elderly smokers after 4 weeks and a significant decrease in the FVC, FEV1/FVC, and PEF after 6 weeks. On balance, the effects of the training persisted 6 weeks after suspending the training only in the FEV1 and VC.

The final inspiratory capacity and ventilation capacity of patients with COPD were reported to be increased after pursed-lip breathing training(14, 20). An at-home breathing rehabilitation program, which consisted of 20 sets of pursed-lip breathing training three times a day, improved the pulmonary function and decreased the occurrence of dyspnea among elderly communitydwelling COPD patients, resulting in better performance of daily activities of living (21).

Pursed lip breathing training involves the use of expiration to improve the pulmonary function. Balloon blowing, as applied in this study, is an exhalation related exercise. During balloon blowing, pursed lip breathing can increase the ventilation capacity of the lungs by increasing the volume of the lungs. The resulting improvement in expiration abilities can help to prevent respiratory disease and improve breathing functions among elderly people. Balloon blowing can also improve the cough reflex, and this can aid the removal of secretions from the respiratory tract.

A previous study measured by Respifit $S^{\circledR}$ (Biegler Gmbh, Austria) the pulmonary function of elderly people following $30 \mathrm{~min}$ diaphragmatic breathing training and inspiratory muscle breathing training, performed three times a week for 8 weeks(22). In that study, the patients' FVC and FEV1 significantly increased, which led to improvements in the respiratory muscles and the elasticity of the chest wall. These results imply that combining a breathing rehabilitation treatment using the breathing apparatus in this study with diaphragmatic breathing training will offer the most benefit in terms of increased pulmonary function. Breathing rehabilitation among elderly subjects can also be expected to have other positive outcomes, such as reducing physical fatigue. It can also play a role in preventing and treating respiratory disease by improving respiratory muscle strength and expiration ability.

Elderly people have a greater risk of lung diseases due to aged related declines in pulmonary function and immunity, in addition to physical and physiological changes and functional deterioration of the respiratory system(23). Among elderly smokers, respiratory disease is a risk factor for decreased pulmonary function(11).

People today are living longer than ever before, and the aging population is experiencing various problems, such as declines in physical and psychological functions, social isolation, and dramatically increased medical expenses(24). Breathing training can be implemented in the elderly to address such problems. However, many rehabilitation hospitals in Korea face time limitations in reality(16).

A breathing rehabilitation program for elderly people aimed at overcoming smoking related increases in respiratory disease, breathing problems, and respiratory muscle weakness needs to be developed.

\section{CONCLUSION}

In conclusion, FBT and BBT had a positive impact on the pulmonary function and minor respiratory muscles of elderly smokers. An at home breathing rehabilitation program, in addition to balloon blowing, could increase the breathing performance of elderly people. It could also diminish dyspnea and improve their physical functioning, resulting in a higher quality of life. 


\section{REFERENCES}

1. Kim YS. The effect of the combined exercise on the risk factors for health related physical fitness, blood lipids, and cardiovascular diseases in the elderly women. Kangnung National University, Master' s thesis 2005.

2. Mayer F, Scharhag-Rosenberger F, Carlsohn A, Cassel M, Müller S, Scharhag J. The intensity and effects of strength training in the elderly. Deutsches Ärzteblatt International 2011; 108(21): 59.

3. Katsiaras A, Newman AB, Kriska A, Brach J. Krishnaswami S, Feingold E, Goodpaster BH. Skeletal muscle fatigue, strength, and quality in the elderly: the Health ABC Study. Journal of Applied Physiology 2005; 99(1): 210-216.

4. Bonomo L, Larici AR, Maggi F, Schiavon F, Berletti R. Aging and the respiratory system. Radiol Clin North Am 2008; 46(4):685-702.

5. Mahler DA, Rosiello RA, Loke J. The aging lung. Clin Geriatr Med 1986; 2(2): 215-225.

6. Chien MY, Kuo HK, Wu YT. Sarcopenia, cardiopulmonary fitness, and physical disability in community-dwelling elderly people. Physical therapy 2010; 90(9):1277-1287.

7. Cha KS. A comparison of pulmonary function, aerobic power, blood pressure, blood hemoglobin in smokers and non-smokers. Korean journal of physical eduaction 2001; 40(3): 845-857.

8. Mokdad AH, Marks JS, Stroup DF, Gerberding JL. Actual causes of death in the United States, 2000. JAMA 2004; 291(10):1238-1245.

9. Shaw M, Mitchell R,Dorling D. Time for a smoke? One cigarette reduces your life by 11 minutes. BMJ 2000; 320(7226):53-53.

10. Mozaffarian D, Kumanyika SK, Lemaitre RN, Olson JL., Burke GL., Siscovick DS. Cereal, fruit, and vegetable fiber intake and the risk of cardiovascular disease in elderly individuals. JAMA 2003; 289(13):1659-1666.

11. Kim EH. Characteristics of respiration and phonation depending of smoking or non smoking by practical musicology students and general male students. Myongjy University, Master' s thesis 2014.

12. Kido S, Nakajima Y, Miyasaka T, Maeda Y, Tanaka T, Yu W, Takayanagi K. Effects of combined training with breathing resistance and sustained physical exertion to improve endurance capacity and respiratory muscle function in healthy young adults. J Phys Ther Sci, 2013 25(5):605-610.
13. Koppers RJ, Vos PJ, Boot CR, Folgering HTM. Exercise performance improves in patients with COPD due to respiratory muscle endurance training. CHEST 2006; 129(4):886-892.

14. Bianchi R, Gigliotti F, Romagnoli I, Lanini B, Castellani C, Grazzini M, Scano G. Chest wall kinematics and breathlessness during pursed-lip breathing in patients with COPD. CHEST, 2004 125(2):459-465.

15. Kim JS, Lee YS. Effects of a Balloon-Blowing Exercise on Lung Function of Young Adult Smokers. Journal of Physical Therapy Science 2012; 24(6):531-534.

16. Seo KC. The Effect of Pulmonary Function and Respiratory Muscle Activity in the Stroke Patients after Complex Breathing Exercise. Daegu University, Master' s thesis 2012.

17. Kim DS. The Relationship of Age, Weight, Height and Smoking History with Spirometric Parameters in Healthy Adults. Bulleun of Dongnam Health College 2003; 21(2):429-440

18. Scherer TA, Spengler CM, Owassapian D, MHOF $\mathrm{E}$, Boutellier URS. Respiratory muscle endurance training in chronic obstructive pulmonary disease: impact on exercise capacity, dyspnea, and quality of life. American Journal of Respiratory and Critical Care Medicine 2000; 162(5):1709-1714.

19. Lee JH. The Effect of Pulmonary Function in the Stroke Patients after feedback breathing exercise. Daegu University, Master' s thesis 2008.

20. Ugalde V, Breslin EH, Walsh SA, Bonekat HW, Abresch RT, Carter GT. Pursed lips breathing improves ventilation in myotonic muscular dystrophy. Archives of physical medicine and rehabilitation 2000; 81(4):472-478.

21. Kim SH. The effect of home-based pulmonary rehabilitation program in COPD patients. Yonsei University, Master' s thesis 2002.

22. Shin HJ. The Effects of respiratory training programs on respiratory function and fatigue of elderly in a facility. Yongin doctorial thesis 2012.

23. Ryu SJ. Pulmonary functions of Elderly inpatients in One Geriatric Hospital in Gwangju area and Efficacy of Pulmonary. Chonnam National University, Master' s thesis 2009.

24. Jin MH. A Study about Influence that have an effect of abdominal breathing on Life Satisfaction in elderly with Dementia. Dongguk doctorial thesis 2013.

25. Shumway-Cook A, and Wollacott MH. Motor Control: Translating Research into Clinical Practice(4ed). Lippincott Williams \& Wilkins, 2011. 
26. Kaminski TW, Perrin DH, Gansneder BM. Eversion strength analysis of uninjured and functionally unstable ankles. J Athl Train 1999; 34(3): 239-245.

27. Lamontagne A, Malouin F, Richards CL, Dumas F. Mechanisms of disturbed motor control in ankle weakness during gait after stroke. Gait Posture 2002; 15(3): 244-255.

28. Kim MJ. Effects of ankle muscle strengthening for preventing of falls in the elderly persons. Korean National University of Physical Education 2001.

29. Menant JC, Steele JR, Menz HB, Munro BJ, Lord SR. Optimizing footwear for older people at risk of falls. J Rehabil Res Dev 2008; 45(8): 1167-1181.

30. Alfieri FM1, Riberto M, Gatz LS, Ribeiro CP, Lopes JA, Battistella LR. Comparison of multisensory and strength training for postural control in the elderly. Clin Interv Aging 2012; 7: 11925.

31. Roll JP, Vedel JP, Ribot E. Alteration of proprioceptive message induced by tendon vibrationinman: A microneurographicstudy. Exp Brain 1989; 76: $213-222$.
32. Verschueren SM, Swinnen SP, Desloovere K, Duysens J. Vibration-induced changes in EMG during human locomotion. J Neurophysiol 2003; 89(3): 1299-1307.

33. Rittweger J, Mutschelknauss M, Felsenberg D. Acute changes in neuromuscular excitability after exhaustive whole body vibration exercise as compared to exhaustion by squatting exercise. Clin Physiol Funct Imaging 2003; 23(2): 81-86.

34. Priplata AA, Patritti BL, Niemi JB, Hughes R, Gravelle DC, Lipsitz LA, Veves A, Stein J, Bonato $\mathrm{P}$, Collins JJ. Noise-enhanced balance control in patients with diabetes and patients with stroke. Ann Neurol 2006; 59(1): 4-12.

35. Shin SS. The Effect of whole body vibration with weighted vest on body composition, knee extensor strength, static standing balance and bone mineral density for postmenopausal wowen. Unpublished doctoral dissertation, Sahmyook University 2012.

36. Torvinen S, Kannu P, Sievänen H, Järvinen TA, Pasanen M, Kontulainen S, Järvinen TL, Järvinen M, Oja P, Vuori I. Effect of a vibration exposure on muscular performance and body balance. Randomized cross-over study. Clin Physiol Funct Imaging 2002; 22(2): 145-152. 